\title{
A Prothrombotic Score Based on Genetic Polymorphisms of the Hemostatic System Differs in Patients with Ischemic Stroke, Myocardial Infarction, or Peripheral Arterial Occlusive Disease
}

\author{
Juliane Herm ${ }^{1,2 t}$, Berthold Hoppe ${ }^{3,4 t}$, Bob Siegerink ${ }^{2}$, Christian H. Nolte ${ }^{1,2,5}$, \\ Jürgen Koscielny ${ }^{6}$ and Karl Georg Haeusler ${ }^{1,2 *}$
}

OPEN ACCESS

Edited by:

Pietro Enea Lazzerini,

University of Siena, Italy

Reviewed by:

Nazareno Paolocci,

Johns Hopkins University,

United States

Kumaravelu Jagavelu,

Central Drug Research Institute, India

*Correspondence:

Karl Georg Haeusler

georg.haeusler@charite.de

these authors have contributed equally to this work.

Specialty section:

This article was submitted to General Cardiovascular Medicine,

a section of the journal

Frontiers in Cardiovascular Medicine

Received: 27 February 2017

Accepted: 24 May 2017

Published: 09 June 2017

Citation:

Herm J, Hoppe B, Siegerink B,

Nolte $\mathrm{CH}$, Koscielny $\mathrm{J}$ and Haeusler KG (2017) A Prothrombotic

Score Based on Genetic

Polymorphisms of the Hemostatic System Differs in Patients with

Ischemic Stroke, Myocardial Infarction, or Peripheral Arterial Occlusive Disease.

Front. Cardiovasc. Med. 4:39. doi: 10.3389/fcvm.2017.00039
${ }^{1}$ Department of Neurology, Charité - Universitätsmedizin Berlin, Berlin, Germany, ${ }^{2}$ Center for Stroke Research Berlin, Charité - Universitätsmedizin Berlin, Berlin, Germany, ${ }^{3}$ Institute of Laboratory Medicine and Pathobiochemistry, Charité Universitätsmedizin Berlin, Berlin, Germany, ${ }^{4}$ Institute of Laboratory Medicine, Unfallkrankenhaus Berlin, Berlin, Germany, ${ }^{5}$ Berlin Institute of Health, Berlin, Germany, ${ }^{6}$ Institute of Transfusion Medicine, Charité - Universitätsmedizin Berlin, Berlin, Germany

Background: While twin studies indicate a genetic component in arterial thrombosis such as ischemic stroke, myocardial infarction (MI), or peripheral arterial occlusive disease (PAOD), the clinical relevance of hemostatic polymorphisms in arterial thrombosis is a matter of debate.

Methods: We analyzed the prevalence of 13 hemostatic polymorphisms [PAI-1, PLAT, F5 (including factor $V$ Leiden and HR2 haplotype), F2, F7, F13A, FGB, TFPI, THBD, MTHFR, ACE, and ITGA2] in patients referred to a tertiary referral center. A "prothrombotic score" was calculated by dividing the number of risk-increasing polymorphisms for thrombosis minus the number of risk-lowering polymorphisms ( $F 7$ and $F 13 A$ ) by the number of polymorphisms tested.

Results: Datasets of 144 patients with prior ischemic stroke (mean age $44 \pm 13$ years; $65 \%$ female) were compared to 62 patients with MI or PAOD (mean age $54 \pm 14$ years; $47 \%$ female). The prothrombotic score was lower in MI and PAOD patients compared to stroke patients [odds ratios 2.7 (95\% confidence intervals 1.1-6.2)]. Frequencies of individual polymorphisms did not differ between both groups.

Conclusion: Patients with Ml or PAOD had a lower burden of prothrombotic mutations compared to patients with prior stroke, indicating that a prothrombotic state might play a different role in distinct forms of arterial thrombosis.

Keywords: thrombophilia, ischemic stroke, myocardial infarction, PAOD, prothrombotic state

Abbreviations: $A C E$, angiotensin converting enzyme; $F$, factor; F5, factor V Leiden; ITGA2, glycoprotein Ia/IIa; MTHFR, methylenetetrahydrofolate reductase; MI, myocardial infarction; OR, odds ratio; PAOD, peripheral arterial occlusive disease; PAI-1, plasminogen activator inhibitor type I; F2, prothrombin; FGB, $\beta$-fibrinogen; THBD, thrombomodulin; PLAT, tissue plasminogen activator; TFPI, tissue-factor-pathway-inhibitor; VTE, venous thromboembolic events. 


\section{INTRODUCTION}

Over the last years, a variety of gene polymorphisms with potential influence on the hemostatic system were described, which disturb the balance of the hemostatic system, including procoagulatory, anticoagulatory as well as fibrinolytic and antifibrinolytic pathways $(1,2)$. Carriership of some polymorphisms is linked to thrombophilia-an increased clotting propensity that is associated with an increased risk of venous thromboembolic events (VTE). However, whether these polymorphisms are also accompanied by an increased risk of arterial thrombosis such as stroke, myocardial infarction (MI), or peripheral arterial occlusive disease (PAOD), or a recurrence thereof, is still a matter of debate $(1,3,4)$.

While the clinical relevance of factor V Leiden (F5) (leading to hypercoagulability due to a reduced ability of factor $\mathrm{V}$ variant degradation) and prothrombin (F2) 20210G > A mutation (leading to increased levels of prothrombin possibly due to increased pre-mRNA stability) has been extensively characterized, the effect of other so-called thrombophilic risk factors, e.g., affecting $\beta$-fibrinogen $(F G B)$ or tissue-factor-pathway-inhibitor (TFPI) is largely unknown. For FGB, an effect on the synthesis rate of fibrinogen is assumed. Genetic predisposition is determined by multiple genetic variations. Thus, a comparison of single genetic variants in often small study populations is likely to be impaired by a varying distribution of other contributing polymorphisms, low penetrance, and low relative risks (5). Moreover, therapeutic implications of genetic subtyping are limited. Subsequently, current guidelines do not support the routine analysis of genetic polymorphisms in patients with arterial thrombosis $(6,7)$. Possibly, a combination or load of different genetic polymorphisms is more informative. This introduces the concept of comparing the overall prothrombotic predisposition-as previously used in venous thrombosis-in distinct cardiovascular diseases (8). Previous research indicates that hypercoagulability might play a different role in different subforms of arterial thrombosis (9). A recent meta-analysis suggests that a prothrombotic state has a greater impact on the risk of ischemic stroke compared to MI (10). Still, these results are based on factorby-factor comparisons, ignoring that the sum of several procoagulant traits will convey a stronger clotting propensity. It is unknown to what extent a genetic score summarizing the clotting propensity would also be different for these two diseases. Therefore, we set out to determine whether such a score is differentially distributed in ischemic stroke patients compared to patients with MI or PAOD referred for thrombophilia testing.

\section{MATERIALS AND METHODS}

\section{Study Design and Study Population}

This analysis was approved by the Ethics Committee of the Charité-Universitätsmedizin Berlin, Germany, and all patients gave informed consent for DNA isolation before genetic testing. We analyzed medical records of 144 patients with ischemic stroke and of 62 patients with either MI $(n=44)$ or PAOD $(n=18)$. These patients had been referred to the Institute of Transfusion Medicine, Charité-Universitätsmedizin Berlin, for thrombophilia testing. The following information was assessed from medical records: patients' age, sex, cardiovascular risk factors, and history of VTE.

\section{Assessment of Genetic Polymorphisms}

All patients in this study were referred for testing for hereditary thrombophilia, which is part of routine care performed at the Institute of Transfusion Medicine, Charité-Universitätsmedizin Berlin, Germany. Heterozygous and homozygous variants of the following gene polymorphisms were distinguished from wild type: plasminogen activator inhibitor type I (PAI-1) [-675 insertion/deletion $(5 \mathrm{G} / 4 \mathrm{G})$ ], tissue plasminogen activator (PLAT) (intron h deletion/insertion), F5 (1691G $>$ A) (Leiden) and HR2 haplotype (4070A>G), F2 (20210G>A), FGB (455G>A), TFPI $(536 \mathrm{C}>\mathrm{T})$, thrombomodulin $(T H B D)(127 \mathrm{G}>\mathrm{A})$, FVII $(F 7)$ (R353Q), FXIII (F13A) (Val34Leu), methylenetetrahydrofolate reductase $(M T H F R)(677 \mathrm{C}>\mathrm{T})$, angiotensin converting enzyme (ACE) (intron 16 insertion/deletion), and glycoprotein Ia/IIa (ITGA2) $(807 \mathrm{C}>\mathrm{T})$.

Genomic DNA was extracted from whole blood using GenoPrepTM Cartridges B and the GenoMTM-6 system (GenoVision, Vienna, Austria). DNA samples were analyzed by an amplification refractory mutation system using allele-specific primer pairs as previously described (11). All primers were synthesized by TIB Molbiol (Berlin, Germany). The polymerase chain reaction was carried out in a thermocycler (GeneAmp PCR System 9700, Applied Biosystems, Darmstadt, Germany) using the following temperature profiles: denaturation $\left(96^{\circ} \mathrm{C}\right.$ for $\left.2 \mathrm{~min}\right)$, followed by 10 cycles $\left(96^{\circ} \mathrm{C}\right.$ for $15 \mathrm{~s}, 65^{\circ} \mathrm{C}$ for $\left.60 \mathrm{~s}\right)$ and 20 cycles $\left(96^{\circ} \mathrm{C}\right.$ for $10 \mathrm{~s}, 61^{\circ} \mathrm{C}$ for $50 \mathrm{~s}$, and $72^{\circ} \mathrm{C}$ for $\left.30 \mathrm{~s}\right)$. Amplification products were separated using electrophoresis and made visible using electrophoresis and UV transillumination.

\section{Prothrombotic Score}

To assess the overall burden of thrombophilia per patient, a prothrombotic score was calculated dividing the number of riskincreasing polymorphisms (heterozygote or homozygote) minus the number of risk-lowering polymorphisms (heterozygote or homozygote) by the number of all 13 polymorphisms tested. Variant alleles of F7 and F13A were considered to be protective. This prothrombotic score ranges theoretically between $0 \%$ (none of tested variants are prothrombotic) and 100\% (all of the tested variants are prothrombotic), with the remark that only a subset of values can be observed as a maximum of 13 markers have been tested in these patient groups. The score was categorized in tertiles based on the given distribution in the stroke cohort. Overall, 19 (10.2\%) patients had to be excluded from this analysis because of incomplete data (defined as less than 12 of 13 hemostatic polymorphisms tested). The excluded patients comprised of 14 stroke patients, $3 \mathrm{MI}$ patients, and 2 POAD patients.

\section{Statistical Analysis}

For categorical data, absolute and relative frequencies were computed. In case of continuous variables with nearly normal distribution, the mean, SD, minimal and maximal values, otherwise median, quartiles, minimal and maximal values were determined. The Fisher's exact test was used to compare proportions for dichotomous outcomes between independent 
groups. Distribution of variant alleles was explored comparing carrier vs. non-carrier status by Fisher's exact test. A direct comparison between the MI/PAOD group and the stroke group with regard to the prothrombotic score was performed using binary logistic regression, yielding odds ratios (OR) and corresponding confidence intervals (CI). To do this, we a priori categorized the risk score in tertiles, with the middle category as reference category. Nominal scaled outcomes were analyzed using the Mann-Whitney test.

\section{RESULTS}

\section{Study Cohort and Baseline Characteristics}

Baseline characteristics of stroke patients as well as patients with MI or PAOD are depicted in Table 1. Stroke patients were significantly younger and more often female than MI or PAOD patients. Compared to patients with MI or PAOD, stroke patients suffered less often from diabetes, arterial hypertension, hyperlipoproteinaemia, or coronary arterial disease.

\section{Prothrombotic Score and Prevalence of Variant Alleles in Patients with Ischemic Stroke vs. Patients with MI or PAOD}

In a tertile analysis, a low prothrombotic score (0-31.1\%) was found in $22.3 \%(n=29)$ of stroke patients and $36.8 \%(n=21)$ of MI/PAOD [OR 2.66 (95\% CI 1.14-6.21)] (Table 2), indicating that more MI/PAOD patients have a lower prothrombotic propensity then stroke patients. This difference was also observed if patients with a prior VTE were excluded from the analysis [OR 2.82 (95\% CI 1.17-6.82)].

Comparing the distribution of individual variant alleles in stroke patients (referred to the thrombophilia clinic) to MI/PAOD patients (referred to the same thrombophilia clinic) there was no statistically significant difference across groups (Table 3 ). In more detail, protective variant $F 13 A$ Val34Leu was numerically more frequent in stroke patients than in MI/PAOD patients [ 44.8 vs. 33.1\%; OR 1.58 (95\% CI 0.85-2.94)], whereas F7 R353Q was

TABLE 1 | Baseline data of stroke patients compared to patients with myocardial infarction (MI) or peripheral arterial occlusive disease (PAOD).

\begin{tabular}{lcc}
\hline & $\begin{array}{c}\text { Stroke patients } \\
(\boldsymbol{n}=\mathbf{1 4 4})\end{array}$ & $\begin{array}{c}\text { MI or PAOD } \\
\text { patients }(\boldsymbol{n}=\mathbf{6 2})\end{array}$ \\
\hline Age, years; mean (SD) (range) & $43.6(13.2)(16-75)$ & $54.4(14.3)(24-78)$ \\
Female sex, $n$ (\%)* & $93(64.6)$ & $29(46.8)$ \\
Comorbidities, $n$ (\%) & & \\
Prior ischemic stroke or TIA & $9(6.3)$ & $1(1.6)$ \\
Diabetes mellitus & $7(4.9)$ & $9(14.5)$ \\
Arterial hypertension & & $40(64.5)$ \\
Hyperlipoproteinaemia* & $47(32.6)$ & $35(56.5)$ \\
Coronary artery disease & $50(34.7)$ & $1(5.6)^{\mathrm{a}}$ \\
Atrial fibrillation & $5(3.5)$ & $5(8.1)$ \\
Prior venous thrombotic event & $4(2.8)$ & $8(12.3)$ \\
Tobacco consumption; $n$ (\%) & $13(9.0)$ & $23(37.1)$ \\
\hline
\end{tabular}

Data are given as $n(\%)$ if not stated otherwise.

${ }^{*} p$-Value $<0.05$.

aNumbers given for $P A O D$ patients only. equally distributed [22.8 vs. $20.9 \%$, OR 1.02 (95\% CI 0.49-2.13)]. F5 $1691 \mathrm{G}>\mathrm{A}$ was found in $18.3 \%$ of stroke patients and $14.5 \%$ of MI/PAOD patients [OR 1.32 (95\% CI 0.58-3.01)], and F2 $20210 \mathrm{G}>\mathrm{A}$ was present in $3.5 \%$ of stroke patients and $9.7 \%$ of MI/PAOD patients [OR 0.34 (95\% CI 0.10-1.17)]. TFPI 536C > T as well as THBD $127 \mathrm{G}>\mathrm{A}$ was found in one stroke patient $(0.7 \%)$ and one MI/PAOD patient (1.6\%). These results show that the prevalence of single polymorphisms was too low to draw strong conclusions about these individuals.

\section{DISCUSSION}

In our study of patients referred for thrombophilia testing, the distribution of the prothrombotic propensity score differed between patients with stroke compared to those with MI or PAOD, while the individual variant alleles of this score did not differ significantly in these cohorts.

Ischemic stroke, MI, and PAOD are widespread diseases with tremendous socioeconomic impact $(12,13)$ and share similar cardiovascular risk factors. While MI and PAOD are (mainly) caused by atherosclerosis, stroke etiology remains undefined ("cryptogenic") in about $25 \%$ of all stroke patients (14). Interestingly, published studies give reason to postulate that a prothrombotic state may play an important role in the pathophysiology of ischemic stroke $(9,10)$.

Our results show that the prothrombotic score (calculated as percentage of risk alleles per subject) - being the overall view of the prothrombotic predisposition of a patient-indeed provides different information. This approach has been used before to assess the risk of (recurrent) VTE and demonstrated a stronger association compared to individual genetic variants $(5,8)$.

Our results indicate that patients referred to a thrombophilia clinic because of MI/PAOD were more likely to have a low vs. normal thrombotic risk score than patients with ischemic stroke (OR of 2.7) (Table 2). This is in line with the hypothesis that there might be a different-and possibly additive-effect of the amount of prothrombotic genetic variants with regard to the risk of MI/PAOD or ischemic stroke, respectively.

These findings lead to the idea that a prothrombotic state may play a role in the pathophysiology of (cryptogenic) stroke (15). This is backed by the RATIO study, which evaluated up to 29 individual markers (including some genetic markers as F5 1691G>A, F2 20210G > A, MTHFR 677C > T, and others) and concluded that hypercoagulability is a risk factor of stroke but not MI in women aged $<50$ years (9). While our findings support this concept, our study was not designed to investigate the pathophysiology of variant forms of arterial thrombosis. Yet, our findings do show

\begin{tabular}{|c|c|c|c|}
\hline $\begin{array}{l}\text { Prothrombotic } \\
\text { score (\%) }\end{array}$ & $\begin{array}{l}\text { Stroke } \\
n(\%)\end{array}$ & $\begin{array}{c}\text { MI/PAOD } \\
n(\%)\end{array}$ & $\begin{array}{c}\text { Odds ratios }(95 \% \\
\text { confidence intervals) }\end{array}$ \\
\hline $0-31.1$ & $29(22.3)$ & $21(36.8)$ & $2.66(1.14-6.21)$ \\
\hline $31.2-38.5$ & $44(33.8)$ & $12(21.1)$ & 1 \\
\hline $38.6-100$ & 57 (43.8) & $24(42.1)$ & $1.54(0.70-3.43)$ \\
\hline
\end{tabular}


TABLE 3 | Prevalence of gene polymorphisms in ischemic stroke patients ( $n=144)$, patients with myocardial infarction (MI) or peripheral arterial occlusive disease (PAOD) $(n=62)$.

\begin{tabular}{|c|c|c|c|c|c|}
\hline Polymorphism (mutation) & Group & $n$ & Heterozygote \% (n) & Homozygote \% (n) & Odds ratios ( $95 \%$ confidence intervals) \\
\hline \multirow[t]{2}{*}{$F G B(-455 G>A)$} & Stroke & 132 & $37.9(50)$ & $2.3(3)$ & $1.34(0.70-2.57)$ \\
\hline & MI/PAOD & 57 & $26.3(15)$ & $7.0(4)$ & \\
\hline \multirow[t]{2}{*}{$\operatorname{TFPI}(536 \mathrm{C}>\mathrm{T})$} & Stroke & 142 & $0.7(1)$ & $0(0)$ & a \\
\hline & MI/PAOD & 62 & $1.6(1)$ & $0(0)$ & \\
\hline \multirow[t]{2}{*}{$T H B D(127 G>A)$} & Stroke & 142 & $0.7(1)$ & $0(0)$ & a \\
\hline & MI/PAOD & 62 & $1.6(1)$ & $0(0)$ & \\
\hline \multirow[t]{2}{*}{ F7 (R353Q) } & Stroke & 132 & $20.5(27)$ & $2.3(3)$ & $1.02(0.49-2.13)$ \\
\hline & MI/PAOD & 58 & $17.7(11)$ & $3.2(2)$ & \\
\hline \multirow[t]{2}{*}{ F13A (Val34Leu) } & Stroke & 143 & $36.4(52)$ & $8.4(12)$ & $1.58(0.85-2.94)$ \\
\hline & MI/PAOD & 62 & $25.8(16)$ & $8.1(5)$ & \\
\hline \multirow[t]{2}{*}{ PAl-1 (-675 I/D) } & Stroke & 142 & $49.3(70)$ & $30.3(43)$ & $1.36(0.67-2.73)$ \\
\hline & MI/PAOD & 62 & $38.7(24)$ & $35.5(22)$ & \\
\hline \multirow[t]{2}{*}{ PLAT (intron h D/l) } & Stroke & 143 & $52.4(75)$ & $34.3(49)$ & $2.08(0.98-4.43)$ \\
\hline & MI/PAOD & 62 & $54.8(34)$ & $21.0(13)$ & \\
\hline \multirow[t]{2}{*}{$F 2(20210 G>A)$} & Stroke & 141 & $3.5(5)$ & $0(0)$ & $0.34(0.10-1.17)$ \\
\hline & MI/PAOD & 62 & $9.7(6)$ & $0(0)$ & \\
\hline \multirow[t]{2}{*}{$F 5(1691 \mathrm{G}>A)$} & Stroke & 142 & $17.6(25)$ & $0.7(1)$ & $1.32(0.58-3.01)$ \\
\hline & MI/PAOD & 62 & $12.9(8)$ & $1.6(1)$ & \\
\hline \multirow[t]{2}{*}{ F5 (4070A>G) } & Stroke & 141 & $12.1(17)$ & $1.4(2)$ & $1.05(0.43-2.55)$ \\
\hline & MI/PAOD & 62 & $12.9(8)$ & $0(0)$ & \\
\hline \multirow[t]{2}{*}{$A C E$ (intron 16 I/D) } & Stroke & 142 & $50.7(72)$ & $19.4(28)$ & $0.59(0.29-1.22)$ \\
\hline & MI/PAOD & 62 & $46.8(29)$ & $33.9(21)$ & \\
\hline \multirow[t]{2}{*}{ MTHFR $(677 \mathrm{C}>\mathrm{T})$} & Stroke & 142 & $41.5(59)$ & $10.6(15)$ & $1.41(0.77-2.57)$ \\
\hline & MI/PAOD & 62 & $33.9(21)$ & $9.7(6)$ & \\
\hline \multirow[t]{2}{*}{ ITGA2 (807C> T) } & Stroke & 130 & $50.0(65)$ & $8.5(11)$ & $0.74(0.39-1.43)$ \\
\hline & MI/PAOD & 55 & $50.9(28)$ & $14.5(8)$ & \\
\hline
\end{tabular}

${ }^{a}$ Analysis was not performed due to the low prevalence.

that the here proposed prothrombotic score-as a way to better assess the prothrombotic propensity instead of studying single factors-does potentially provide this information in more detail than an array of individual markers in patients with different types of arterial thrombosis. However, further studies are needed to evaluate this hypothesis.

\section{Limitations}

Some limitations have to be taken into account in the interpretation of these results. First, the sample size is limited, as we included only patients referred to a tertiary referral center for genetic testing on the presence of thrombophilia. Thus, a potential referral bias has to be taken into account. This is a major problem of similar studies, which use population or healthy controls. However, we believe that a potential referral bias is minimized in the present analysis, as the reason for referral is assumed to be similar for both groups. Furthermore, the lower number of patients with MI or PAOD limits the power of our analysis. Moreover, combining MI and PAOD patients for analysis might have introduced bias as one of the two combined groups might have a different distribution. However, this bias would dilute any true association, rendering the reported odds ratio of 2.7 an underestimation of the real association. Moreover, stroke is a heterogeneous disease, and a prothrombotic state may play a stronger role in (young) stroke patients without or with just a few established cardiovascular risk factors. Of note, stroke patients referred for thrombophilia testing do not represent the overall cohort of stroke patients. As follow-up data were not available, we cannot answer the question whether risk assessment by routine genetic testing can reduce recurrence of arterial thrombosis. Finally, by reporting a wide variety of gene polymorphisms, not all polymorphisms were analyzed in every single patient with stroke, MI, or PAOD, respectively.

\section{CONCLUSION}

The bigger picture of prothrombotic predisposition needs to be assessed instead of focusing on individual variant alleles. Our data indicate a different impact of hereditary thrombophilia on the risk of ischemic stroke compared to the risk of MI or PAOD. Large studies in well-defined cohorts of stroke, MI, and PAOD patients are needed to better understand the reported differences and the clinical relevance of a genetic prothrombotic score.

\section{ETHICS STATEMENT}

This analysis was approved by the Ethics Committee of the Charité-Universitätsmedizin Berlin, Germany (EA1/048/11). As data was obtained retrospectively, consent to participate was not obtained. 


\section{AUTHOR CONTRIBUTIONS}

$\mathrm{KGH}$ and $\mathrm{BH}$ conceived the study. $\mathrm{KGH}$ supervised data assessment, wrote and drafted the manuscript together with $\mathrm{JH}$. JH also performed statistical analysis. BS made substantial contributions to data analysis, supervised statistical analysis, and critically revised the manuscript. $\mathrm{BH}$, $\mathrm{JK}$, and $\mathrm{CN}$ critically revised the manuscript for important intellectual content. All the authors read and approved the final manuscript.

\section{REFERENCES}

1. Casas JP, Hingorani AD, Bautista LE, Sharma P. Meta-analysis of genetic studies in ischemic stroke: thirty-two genes involving approximately 18,000 cases and 58,000 controls. Arch Neurol (2004) 61:1652-61. doi:10.1001/archneur. 61.11 .1652

2. Hankey GJ, Eikelboom JW. Editorial comment - routine thrombophilia testing in stroke patients is unjustified. Stroke (2003) 34:1826-7. doi:10.1161/01. STR.0000083533.81284.0B

3. Morris JG, Singh S, Fisher M. Testing for inherited thrombophilias in arterial stroke: can it cause more harm than good? Stroke (2010) 41:2985-90. doi:10.1161/STROKEAHA.110.595199

4. Boekholdt SM, Kramer MHH. Arterial thrombosis and the role of thrombophilia. Semin Thromb Hemost (2007) 33:588-96. doi:10.1055/s-2007-985755

5. van Hylckama Vlieg A, Baglin CA, Bare LA, Rosendaal FR, Baglin TP. Proof of principle of potential clinical utility of multiple SNP analysis for prediction of recurrent venous thrombosis. J Thromb Haemost (2008) 6:751-4. doi:10.1111/j.1538-7836.2008.02920.x

6. Ye Z, Liu EHC, Higgins JPT, Keavney BD, Lowe GDO, Collins R, et al. Seven haemostatic gene polymorphisms in coronary disease: meta-analysis of 66,155 cases and 91,307 controls. Lancet (2006) 367:651-8. doi:10.1016/ S0140-6736(06)68263-9

7. Feinbloom D, Bauer KA. Assessment of hemostatic risk factors in predicting arterial thrombotic events. Arterioscler Thromb Vasc Biol (2005) 25:2043-53. doi:10.1161/01.ATV.0000181762.31694.da

8. de Haan HG, Bezemer ID, Doggen CJM, Le Cessie S, Reitsma PH, Arellano AR, et al. Multiple SNP testing improves risk prediction of first venous thrombosis. Blood (2012) 120:656-63. doi:10.1182/blood-2011-12-397752

9. Siegerink B, Maino A, Algra A, Rosendaal FR. Hypercoagulability and the risk of myocardial infarction and ischemic stroke in young women. J Thromb Haemost (2015) 13:1568-75. doi:10.1111/jth.13045

10. Maino A, Rosendaal FR, Algra A, Peyvandi F, Siegerink B. Hypercoagulability is a stronger risk factor for ischaemic stroke than for myocardial infarction: a systematic review. PLoS One (2015) 10:e0133523. doi:10.1371/journal.pone. 0133523

\section{ACKNOWLEDGMENTS}

The authors thank Julia Herde (CSB) for critically revising the manuscript.

\section{FUNDING}

The project has received funding from the German Federal Ministry of Education and Research via the grant Center for Stroke Research Berlin (01 EO 0801).

11. Hoppe B, Heymann GA, Koscielny J, Hellstern P, Kiesewetter H, Salama A. Screening for multiple hereditary hypercoagulability factors using the amplification refractory mutation system. Thromb Res (2003) 111:115-20. doi:10.1016/j.thromres.2003.08.024

12. Schmid T. Costs of treating cardiovascular events in Germany: a systematic literature review. Health Econ Rev (2015) 5:27. doi:10.1186/s13561-015-0063-5

13. Jennum P, Iversen HK, Ibsen R, Kjellberg J. Cost of stroke: a controlled national study evaluating societal effects on patients and their partners. BMC Health Serv Res (2015) 15:466. doi:10.1186/s12913-015-1100-0

14. Hart RG, Diener H-C, Coutts SB, Easton JD, Granger CB, O'Donnell MJ, et al. Embolic strokes of undetermined source: the case for a new clinical construct. Lancet Neurol (2014) 13:429-38. doi:10.1016/S1474-4422(13)70310-7

15. Haeusler KG, Herm J, Hoppe B, Kasabov R, Malzahn U, Endres M, et al. Thrombophilia screening in young patients with cryptogenic stroke. Prevalence of gene polymorphisms compared to healthy blood donors and impact on secondary stroke prevention. Hamostaseologie (2012) 32:147-52. doi:10.5482/ha-1175

Conflict of Interest Statement: KGH reports lecture fees and (a) study grant(s) by Bayer Healthcare and Sanofi-Aventis, lecture fees from Boehringer Ingelheim, Pfizer, and Bristol-Myers Squibb as well as advisory board fees from Pfizer, Edwards Lifesciences, Medtronic, and EIP Pharma. CN reports lecture fees and travel support by Bayer Healthcare, Sanofi-Aventis, Bristol-Myers Squibb, Pfizer, and Boehringer Ingelheim. JK reports speaker honoraria from Bayer Healthcare Pharmaceuticals, Boehringer Ingelheim, CSL Behring, Sanofi-Aventis, Pfizer, BMS, Novartis, Aspen, Daiichi Sankyo, and Novo Nordisk. JK is also a medical advisor for CSL Behring International, Bayer HealthCare Pharmaceuticals (national and international), Astra Zeneca (national), and Novo Nordisk (national). JH, BH, and BS report no conflicts of interest.

Copyright (c) 2017 Herm, Hoppe, Siegerink, Nolte, Koscielny and Haeusler. This is an open-access article distributed under the terms of the Creative Commons Attribution License (CC BY). The use, distribution or reproduction in other forums is permitted, provided the original author(s) or licensor are credited and that the original publication in this journal is cited, in accordance with accepted academic practice. No use, distribution or reproduction is permitted which does not comply with these terms. 